\title{
Rich Club Organization and Intermodule Communication in the Cat Connectome
}

\author{
Marcel A. de Reus and Martijn P. van den Heuvel \\ Department of Psychiatry, University Medical Center Utrecht, Brain Center Rudolf Magnus, 3508 GA Utrecht, The Netherlands
}

Macroscopic brain networks have been shown to display several properties of an efficient communication architecture. In light of global communication, the formation of a densely connected neural "rich club" of hubs is of particular interest, because brain hubs have been suggested to play a key role in enabling short communication pathways within neural networks. Here, analyzing the cat connectome as reconstructed from tract tracing data (Scannell et al., 1995), we provide several lines of evidence of an important role of the structural rich club to interlink functional domains. First, rich club hub nodes were found to be mostly present at the boundaries between functional communities and well represented among intermodule hubs, displaying a diverse connectivity profile. Second, rich club connections, linking nodes of the rich club, and feeder connections, linking non-rich club nodes to rich club nodes, were found to comprise $86 \%$ of the intermodule connections, whereas local connections between peripheral nodes mostly spanned between nodes of the same functional community. Third, almost $90 \%$ of all intermodule communication paths were found to follow a sequence or "path motif" that involved rich club or feeder edges and thus traversed a rich club node. Together, our findings provide evidence of the structural rich club to form a central infrastructure for intermodule communication in the brain.

\section{Introduction}

Brain function depends on efficient processing and integration of information within a complex network of neuronal interactions. Studies examining the macroscopic architecture of neural networks have suggested that the mammalian brain, and likely neural systems in general, are organized according to a "small-world modular" architecture, combining high levels of local clustering and a community structure with short global communication pathways (Hagmann et al., 2008; Bullmore and Sporns, 2009; van den Heuvel and Hulshoff Pol, 2010). It has been hypothesized that local clustered communities may ensure segregation and local specialization of the brain, whereas the presence of short communication relays may provide an infrastructure for global integration of information (Sporns et al., 2005; Sporns, 2011). Studies have further noted the existence of a relatively small but crucial set of highly connected regions that play a central role in the overall architecture of brain networks (Hagmann et al., 2008; Buckner et al., 2009; Tomasi and Volkow, 2010; Sepulcre et al., 2012; van den Heuvel et al., 2012). Besides being individually "rich" in connectivity, this set of putative hubs has been suggested

Received April 4, 2013; revised May 17, 2013; accepted June 6, 2013.

Author contributions: M.A.d.R. and M.P.v.d.H. designed research; M.A.d.R. and M.P.v.d.H. performed research; M.A.d.R. and M.P.v.d.H. contributed unpublished reagents/analytic tools; M.A.d.R. and M.P.v.d.H. analyzed data; M.A.d.R. and M.P.v.d.H. wrote the paper.

The authors gratefully thank Olaf Sporns for inspiring discussions and helpful comments, Eus van Someren for helping out with information about cat brain anatomy, and Nadja de Bruin for helping with the schematic drawings (Fig. 1b,c). MPvdH was supported by Netherlands Organization for Scientific Research VENI Grant 451-12-001 and a fellowship of the Brain Center Rudolf Magnus.

Correspondence should be addressed to Martijn van den Heuvel, Department of Psychiatry, University Medical Center Utrecht, Brain Center Rudolf Magnus, Heidelberglaan 100, P0 Box 85500, 3508 GA Utrecht, The Netherlands. E-mail:m.p.vandenheuvel@umcutrecht.nl.

DOI:10.1523/JNEUROSCI.1448-13.2013

Copyright $\odot 2013$ the authors $\quad 0270-6474 / 13 / 3312929-11 \$ 15.00 / 0$ to show a dense level of interconnectivity, suggesting the formation of a "core" or "rich club" in the cat, macaque, and human brain (Zamora-López et al., 2010; van den Heuvel and Sporns, 2011; Harriger et al., 2012; van den Heuvel et al., 2012), as well as in the neural system of the nematode Caenorhabditis elegans (Zamora-López et al., 2010; Towlson et al., 2013). From the perspective of information integration, the formation of a centrally connected rich club is of particular interest, because it may act as a central backbone for global brain communication (ZamoraLópez et al., 2009; Bullmore and Sporns, 2012; van den Heuvel et al., 2012; Collin et al., 2013).

Animal studies allow for a detailed reconstruction of white matter pathways through means of neural tracing of axonal connections (Selemon and Goldman-Rakic, 1988; Felleman and Van Essen, 1991; Scannell et al., 1995). The cat connectome dataset, as presented by Scannell et al. (1995), involves detailed tracing reconstructions of the cat's white matter pathways, including information about the directionality of white matter projections, a property that remains out of reach of present day in vivo neuroimaging techniques. Moreover, in the case of the cat connectome, a priori neurophysiological information about the functional role of each included brain area facilitates the description of four distinct functional communities, independent of the spatial or topological organization of the underlying structural network (Scannell and Young, 1993; Young, 1993; Scannell et al., 1995). Here, reanalyzing this connectivity dataset with network analysis tools, combining information on the functional organization of the cat brain with information about the cat's structural white matter pathways, we show an important role for the structural rich club in forming a central infrastructure that interlinks all communities and shapes intermodule communication pathways in the cat brain. 


\section{Materials and Methods}

\section{Connectivity dataset}

The cat connectivity dataset comprises a description of corticocortical connections in the cat brain as published by Scannell et al. (1995), a connectivity set resulting from a comprehensive literature search of anatomical tracing studies in the cat cortex. No specific information on the sex of the included animals was available, suggesting that the dataset includes combined information on connectivity of male as well as female cats (Scannell et al., 1995). The parcellation scheme as used by Scannell et al., dividing the cat cortex into 65 distinct, non-overlapping regions, was based on previous work considering the cytoarchitecture and physiology of cortical regions and did not involve clear information on subcortical regions of the cat brain. Detailed information on the delineated regions, including information on the used parcellation scheme, abbreviations and possible overlap with other parcellation schemes, as well as information on the physiological characteristics of these regions, is given in the appendix of the original study by Scannell et al. (1995). The connectivity dataset incorporates data of one hemisphere, including 65 regions and 1139 interregional macroscopic axonal projections, with the strength of the connections measured in three levels: 1 , reflecting a weak connection; 2 , reflecting a medium strength connection; and 3, reflecting a strong connection. The connectivity dataset was transformed to a binary (i.e., unweighted) connectivity matrix, including information on all nonzero entries (i.e., all connections with weights ranging from 1 to 3 ) and interpreted as an unweighted graph $G=(V, E)$, with $V$ describing the nodes of the network taken as the collection of 65 cortical regions and $E$ the collection of macroscopic projections between nodes $V$ in the network.

\section{Functional modules}

The cat connectivity dataset included a classification of the cortical regions (i.e., nodes in the network) into four categories as defined by Scannell et al. (1995): (1) visual regions (18 regions); (2) auditory regions (10 regions); (3) somatomotor regions (18 regions); and (4) frontolimbic regions (19 regions). This subdivision was based on neurophysiological information about the functional role of each included brain area. The four categories were taken and interpreted as an a priori classification of the nodes of the cat connectome into four functional communities, as originally proposed by Scannell et al. (1995). Verifying this a priori definition on the basis of the connectivity structure of the network itself (Hagmann et al., 2008; Harriger et al., 2012), using Newman's directed community detection algorithm (Newman, 2006; 1000 runs, taking the solution with the highest modularity score), revealed a highly similar community pattern, with the only clear difference being that the somatomotor community is further subdivided into two separate modules (one module involving regions 17, 18, 19, PMLS, AMLS, 21a, 21b, ALG, 7, and region SVA and the other module involving regions PLLS, ALLS, VLS, DLS, and 20a).

\section{Rich club organization}

Rich club organization of a network describes the phenomenon that high-degree nodes of the network are more densely connected than one would expect on the basis of their individual degree alone (Colizza et al., 2006; for a detailed description of rich club analyses on brain networks, see van den Heuvel and Sporns, 2011; Zamora-López et al., 2009, 2010). In short, for each node $i$ of the cat connectome, its individual in-degree (i.e., the number of incoming connections) and out-degree (i.e., the number of outgoing connections) was computed. For each level of $k$, the subgraph $S_{k}$ consisting of $N_{>k}$ nodes with a combined in-degree and out-degree larger than $k$ was selected. The rich club coefficient $\Phi(k)$ of level $k$ was computed as the ratio between the number of connections $E_{>k}$ present within this subgraph $S_{k}$ and the total number of possible connections in $S_{k}$ [i.e., $N_{>k} \times\left(N_{>k}-1\right)$ ], formally given by Colizza et al. (2006):

$$
\Phi(k)=\frac{E_{>k}}{N_{>k}\left(N_{>k}-1\right)} .
$$

To compensate for the fact that in randomized networks higher-degree nodes also have a higher probability of becoming interconnected, the cat rich club coefficient $\Phi(k)$ was compared with the rich club coefficient of a set of randomized graphs with a similar degree sequence in which the overall global topology was destroyed. To this end, 1000 random graphs were formed, randomly rewiring the connections of the directed connectivity matrix of the cat connectome while preserving the in-degree and out-degree of each node $i$ in the network (i.e., hubs remain hubs), and the rich club coefficient $\Phi_{\text {random }}(k)$ of these random graphs was computed to obtain a null distribution. For each level of $k, \Phi(k)$ was assigned a $p$ value as the percentage of the null distribution that exceeded the rich club coefficient as observed in the original network. A network is said to display a rich club organization if, for a range of $k, \Phi(k)>\Phi_{\text {random }}(k)$, or, expressed differently, if the ratio $\Phi_{\text {norm }}(k)$ between $\Phi(k)$ and $\Phi_{\text {random }}(k)$ exceeds 1.

\section{Rich club selection}

In this study, the cat rich club was taken as the top 15 highest-degree nodes in the network, effectively selecting a rich club threshold of $k>45$ from the range in which $\Phi(k)$ significantly exceeded $\Phi_{\text {random }}(k)$ (see Fig. $1 a)$. Previous studies have demonstrated that very consistent results are found across threshold choices and across rich club selection criteria (Harriger et al., 2012; Van den Heuvel et al., 2013). In this context, it could be noted that, like the small-world phenomenon, the presence or absence of rich club organization should be regarded as a topological property of the network as a whole rather than a property of a single subset of nodes. The existence of a rich club organization in a network is most often displayed by the presence of a "rich club regime," an interval on the degree range for which $\Phi_{\text {norm }}(k)$ significantly exceeds 1 , not only by the presence of a single rich club.

\section{Participation of nodes in functional modules}

Distinct roles can be assigned to the nodes of a network depending on their intermodule and intramodule connectivity profile (Guimerà and Nunes Amaral, 2005; Sporns et al., 2007; Fornito et al., 2012). To this end, two metrics were examined in detail: (1) the within-module degree $z$ score; and (2) the between-module participation coefficient.

Within-module degree z-score. Taking the functional communities as defined by Scannell et al. (1995) as module classification, the withinmodule degree $z$-score (Guimerà and Nunes Amaral, 2005) of node $i$ was computed as follows:

$$
z_{i}=\frac{\kappa_{i S}-\kappa_{S}}{\sigma_{S}}
$$

where $\kappa_{i S}$ is the number of connections of node $i$ to other nodes in the same module $S$, and $\kappa_{S}$ and $\sigma_{S}$, respectively, represent the mean and SD of the within-module degree over all nodes in $S$. As such, $z_{i}$ describes how well connected (on the structural level) node $i$ is to the other nodes in its (functional) module. High within-module degree $z$-scores thus reflect a high level of intramodule connectivity of node $i$ (Guimerà and Nunes Amaral, 2005). For each node, both an in-degree $z_{i}^{\text {in }}$ and out-degree $z_{i}^{\text {out }}$ score was computed, based on the incoming and outgoing connections of a node, respectively.

Between-module participation coefficient. In addition to the withinmodule degree $z$-score, providing information on the level of intramodule connectivity, the between-module participation coefficient $P_{i}$ quantifies the extent to which the connections of a node are evenly distributed across the different functional modules (Guimerà and Nunes Amaral, 2005). It is given by:

$$
P_{i}=1-\sum_{S=1}^{4}\left(\frac{\kappa_{i S}}{k_{i}}\right)^{2},
$$

where $\kappa_{i S}$ is the number of structural links from node $i$ to nodes in functional module $S$, and $k_{i}$ is the total degree of node $i$. As such, $P_{i}$ expresses the role of node $i$ in interconnecting different modules. For each node, both an in-degree $P_{i}^{\text {in }}$ and out-degree $P_{i}^{\text {out }}$ score was computed, based on the incoming and outgoing connections of a node, respectively.

Nodes were classified according to their in-degree and out-degree $z_{i}$ and $P_{i}$ scores. First, for each of the four metrics separately, node values 
were ranked according to their metric levels. Second, across metrics, each node was given a rank score ranging from 0 to 4 , expressing the number of times it ranked among the top 15 nodes on each of the four metrics. Third, inspired by the concept of Guimerà and Nunes Amaral (2005) and Sporns et al. (2007) who labeled nodes that display a high $z_{i}$ (originally defined as $>2.5$ ) and high $P_{i}$ (originally defined as $>0.3$ ) as "connector hubs," nodes that displayed a high ranking score $(\geq 3)$ were defined as "intermodule hubs" to underscore their central character of linking different functional modules.

\section{Centrality}

For each node, the betweenness centrality $B_{i}$ and level of "PageRank" centrality PageRank ${ }_{i}$ (Page et al., 1999) were computed. The level of $B_{i}$ of node $i$ expresses the proportion of all shortest paths between node $j$ and node $h$ in the network that travel through node $i$, summed over all combinations of $h$ and $j$ in the network (with $h \neq j$ ), and provides an estimate of the role of a node in global network communication. The level of PageRank ${ }_{i}$ of a node reflects how frequently node $i$ is visited by a (infinite) random walker (Rubinov and Sporns, 2010), a type of analysis that has been suggested to provide insight into diffusion-like communication processes in a graph (Goñi et al., 2013). Higher levels of PageRank reflect a more central role of a node in the overall network topology.

\section{Overlapping node communities}

To detect regions of overlap between the four a priori defined functional modules of the cat brain, a "fuzzy" overlapping community approach, driven by efficient encoding of random walker trajectories, was applied to the structural connectivity network (for technical details, see Esquivel and Rosvall, 2011). This algorithm allows nodes of the network to participate in multiple communities on top of their original community assignment. This approach thus extends the initial division of the network (i.e., dividing the network into four non-overlapping functional modules) to an overlapping community pattern, resulting in a community pattern in which nodes are allowed to participate in other communities besides their original assignment. Nodes that participate in multiple communities can be interpreted to be on the boundary between modules, taking on a mediating role between the two (or more) communities to which they are assigned (Esquivel and Rosvall, 2011).

\section{Node and connection classes}

Rich club definition allowed for a classification of the nodes of the cat network into rich club and non-rich club nodes. Furthermore, according to the node classification, the connections of the cat connectome were divided into three connection classes: (1) rich club connections, linking rich club members; (2) feeder connections, linking non-rich club nodes to rich club nodes; and (3) local connections, linking non-rich club nodes (van den Heuvel et al., 2012). Including information on the directionality of the connections, feeder connections were further subdivided into feeder-in connections, projecting from non-rich club to rich club nodes, and feeder-out connections, projecting from rich club nodes to non-rich club nodes. In addition, combining connectivity information with information on the functional modules, connections were labeled as intramodular when they connected nodes of the same functional community or intermodular when they connected nodes of different functional communities. Finally, using information on the directionality of the edges, connections were labeled as bidirectional or unidirectional.

\section{Structural homogeneity of connections}

For each connection $c$, the structural homogeneity was computed as the overlap $O$ between the neighbors of its endpoints $i$ and $j$ (i.e., the nodes connected by $c$ ), formally given by the following:

$$
O=\frac{\left|n_{i} \cap n_{j}\right|}{\left|n_{i} \cup n_{j}\right|},
$$

where $n_{i}$ and $n_{j}$ are the collection of neighbors of node $i$ and node $j$, respectively. Higher $O$ values indicate that node $i$ and $j$ have similar neighbors, suggesting that $c$ links nodes with a comparable role in the network.

\section{Module diversity of connections}

The module diversity of a connection $c$ between nodes $i$ and $j$ was defined as the product $p_{i} \times p_{j}$, where $p_{i}$ and $p_{j}$ are equal to the fraction of functional modules that were directly connected to, respectively, node $i$ and node $j$. As such, a high module diversity score reflects that a connection is embedded between two regions in which information from many functional modules might merge, whereas a low diversity score suggests that a connection facilitates the communication of more unimodal information.

\section{Statistical analyses}

Permutation testing was used to assess statistical significance of group differences on node and connection metrics. First, for a given metric (e.g., node metrics such as $P_{i}$ or $B_{i}$ ), the difference between the mean of the two groups (e.g., rich club vs non-rich club) was computed. Second, for $N$ permutations, the metric values were randomly assigned to two random groups (of equal size to the two original classes), and, for each permutation, their group difference was computed. In this study, $N$ was (in all tests) set to 10,000 . This resulted in a distribution of differences that one can expect under the null hypothesis when no statistical difference is present between the two groups. On the basis of this null distribution, the original difference between the two groups was assigned a (one-tailed) $p$ value as the proportion of the null-distribution values that exceeded the observed original difference. Note that, because the cat dataset involves a reconstruction of the cat connectome based on the aggregation of connectivity data across a large number of studies, it does not take into account intersubject variability. The degrees of freedom in all statistical tests thus come from the dimensions of the network and not from the number of subjects.

\section{Path motifs}

The concept of "path motifs" (van den Heuvel et al., 2012) was applied and extended. A path motif of a communication path expresses the order in which the edge categories [i.e., rich club (RC), feeder (F), local (L)] are traversed and thus defines "groups of paths." To this end, all shortest communication paths (including "degenerate paths") in the network were computed (van den Heuvel et al., 2012) and labeled with the categories of connections passed, and the prevalence of each unique sequence was computed (van den Heuvel et al., 2012). Within an (un)weighted network, multiple degenerate paths of equal length between two nodes can exist. Describing paths of minimal distance, referred to as "communication paths," a degenerate path was taken as one that connected node $i$ and node $j$ with equal length, crossing a node and an edge only a single time on its path. The prevalence of each path motif (e.g., L-L, F-F, $\mathrm{F}-\mathrm{RC}-\mathrm{F}$, etc.) was computed as the ratio between the number of times a path motif was present and the total number of examined paths (i.e., including all degenerate paths). Extending previous studies on path motifs in humans (van den Heuvel et al., 2012), macaque cortex (Harriger et al., 2012), and Caenorhabditis elegans (Towlson et al., 2013), path motifs were examined in more detail with edges now labeled not only according to their primary connection class (rich club, feeder, local) but also according to their directional character and their intermodular versus intramodular role.

\section{Comparison with random networks}

Because studies have emphasized the importance of proper normalization of network metrics (Bialonski et al., 2011; Zalesky et al., 2012), the pattern of path motif counts was compared with the pattern of motifs occurring in an ensemble of random networks (van den Heuvel et al., 2012). A set of a 1000 random networks were generated, randomly rewiring the connections of the cat dataset, preserving the in-degree and out-degree of each node but randomizing the overall topology of the network (Maslov and Sneppen, 2002). Because the in-degree and outdegree of each node remained equal to its value in the original cat dataset, node labels (i.e., rich club/non-rich club labels) were also kept equal to the labels in the cat brain, and connections in the random networks were labeled as rich club, feeder, and local connections according to this node classification. Next, of each of the labeled random networks, motif counts (given as proportions of the total number of examined paths) of each of the path motifs as observed in the original cat dataset were com- 

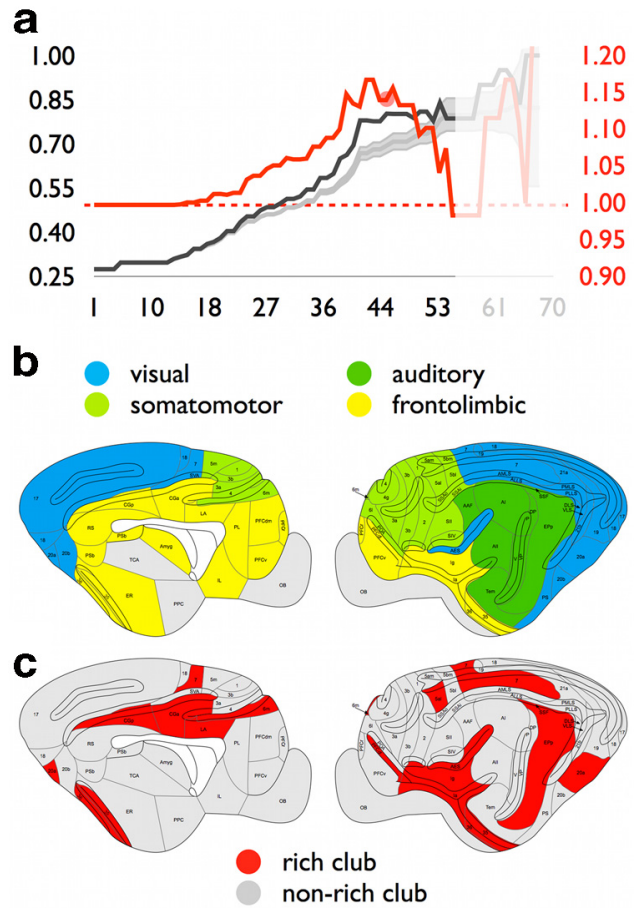

Figure 1. $\quad \boldsymbol{a}$, Rich club curve. $\boldsymbol{a}$ shows the unweighted rich club coefficient $\Phi(k)$ of the cat connectome (black line) for a range of $k=1$ to $k=68$, the mean rich club curve $\Phi_{\text {random }}(k)$ of a set of 1000 comparable random graphs (gray line, randomizing the connections of the cat network while preserving degree sequence), and their ratio (red line). Top and bottom gray lines mark the SD of the rich club coefficient over the 1000 random networks. The red circle indicates the $k>45$ level. $\boldsymbol{b}$ shows a schematic figure of the cat cortex (adapted from Scannell et al., 1995) with regions colored according to their functional community: somatomotor, visual, auditory, and frontolimbic. c shows a regional plot with the rich club members depicted in red and non-rich club nodes depicted in gray.

puted, providing a null distribution of path motif prevalence at chance level. Based on this null distribution, each path motif count as observed in the original dataset was assigned a $p$ value as the number of entries in the null distribution that exceeded the path motif count in the original dataset.

\section{Results}

\section{Rich club nodes and non-rich club nodes}

Confirming previous findings, structural connectivity of the cat connectome showed a rich club organization, with a normalized rich club coefficient $\Phi_{\text {norm }}(k)>1(p<0.05$, Bonferroni's corrected) for a range of $k=15$ to $k=49$ (i.e., the rich club regime; Fig. 1a), consistent with the previous report on rich club organization in the cat connectome of Zamora-López et al. (2009). In this study, from the rich club regime, the rich club was selected as the top 15 (23\%) highest-degree nodes (in-degree and out-degree combined), corresponding with a rich club level of $k>45$. Consistent findings were found when the rich club was selected at different levels (e.g., $k>43$ being the peak of the $\Phi_{\text {norm }}(k)$ curve, or at $k>47)$. At $k>45$, the rich club comprised the regions $20 \mathrm{a}$, 7, AES, SSF, EPp, 6m, 5al, PFCdl, Ia, Ig, CGa, CGp, LA, 35, and 36. The level of rich club density (i.e., the percentage of the total possible number of connections that was present) between these 15 hub nodes was found to be $77 \%$ (1.14 times more than compared with 1000 random networks, $p<0.001)$. A schematic drawing of the regions of the cat cortex, indicating the four functional modules (i.e., visual, auditory, somatomotor, and frontolimbic) and rich club regions is shown in Figure $1, b$ and $c$.
Information on the directionality of the connections allows for an additional generalization of the rich club concept to a specific in-degree and out-degree rich club. Over the network, nodal in-degree and out-degree showed a strong correlation $(r=$ $0.72, p<0.001$, linear regression), indicating that high in-degree nodes also show (on average) a high out-degree. As a result, defining a rich club on the basis of in-degree or out-degree only (selected as the top 15 nodes with highest in-degree or outdegree, respectively) revealed high overlap with the rich club as defined on the combined degree, with 14 of 15 nodes overlapping (20a, 7, AES, EPp, 6m, 5al, PFCdl,Ia, Ig, CGa, CGp, LA, 35, and 36 ) on the basis of in-degree and 11 of 15 nodes overlapping (20a, 7, AES, EPp, 6m, Ia, Ig, CGa, CGp, 35, and 36) on the basis of out-degree. Ten of 15 rich club nodes $(66 \%)$ were revealed to have a higher in-degree than out-degree, in contrast to $33 \%$ of non-rich club nodes.

\section{Participation of rich club nodes in functional modules}

Rich club members were found to be present in all functional modules (Fig. 2a,b), suggesting a diverse connectivity profile of the rich club as a whole. Rich club nodes showed a significantly higher within-module degree $z_{i}^{\text {in }}$ score (mean $\pm \mathrm{SD}$; rich club nodes, $0.43 \pm 0.76$; non-rich club nodes, $-0.13 \pm$ $1.00 ; p=0.044 ; 10,000$ permutations) but not a different $z_{i}^{\text {out }}$ (rich club, $0.028 \pm 0.79$; non-rich club, $-0.01 \pm 1.03 ; p=$ 0.907 ) compared with non-rich club nodes (Fig. $3 b$ ). Furthermore, both the participation coefficient $P_{i}^{\text {in }}$ (rich club, $0.68 \pm$ 0.0337 ; non-rich club, $0.3352 \pm 0.22 ; p<0.001$ ) and $P_{i}^{\text {out }}$ (rich club, $0.65 \pm 0.0611$; non-rich club, $0.3936 \pm 0.21 ; p<0.001$ ) were found to be higher for rich club nodes compared with non-rich club nodes, indicating a diverse connectivity character of rich club nodes.

Obtaining for each node an overall rank score, expressing the number of times a node ranked among the top 15 highest indegree and out-degree $z_{i}$ and $P_{i}$ values, rich club nodes displayed a significant higher ranking score compared with the class of non-rich club nodes (mean $\pm \mathrm{SD}$; rich club nodes, $2.13 \pm 0.83$; non-rich club nodes, $0.56 \pm 0.58 ; p<0.001 ; 10,000$ permutations). The distribution of rank scores across the classes of rich club and non-rich club nodes is shown in Figure $2 c$, showing that all nodes scoring a rank score of 3 (which is the highest ranking score in the network; no nodes showed a score of 4 ) were found to be among rich club nodes, including 6 of the total of 15 rich club nodes. To reflect both their high degree and their intermodular character (all six were among the top $15 P^{\text {in }}$ and $P^{\text {out }}$ scores), these nodes are referred to as intermodule hubs. The level of rich club density (i.e., percentage of possible connections present) between these six intermodule hubs was found to be $87 \%$ (1.16 times more than compared with the random condition; 1000 networks examined). In addition, 11 (73\%) of the 15 rich club nodes showed a score of 2 or more compared with only $5(10 \%)$ of the 50 non-rich club nodes.

\section{Rich club centrality}

Rich club nodes showed a significantly higher betweenness centrality $B_{i}$ (mean $\pm \mathrm{SD}, 0.041 \pm 0.025$ ) compared with the group of non-rich club nodes $(0.006 \pm 0.0049 ; p<0.001 ; 10,000$ per-

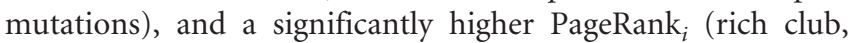
$0.02 \pm 0.0085$; non-rich club, $0.013 \pm 0.0038 ; p<0.01$ ), indicating a central role of rich club nodes in the overall network structure. $B_{i}$ and PageRank ${ }_{i}$ values and their interaction are shown in Figure 3 . 
a
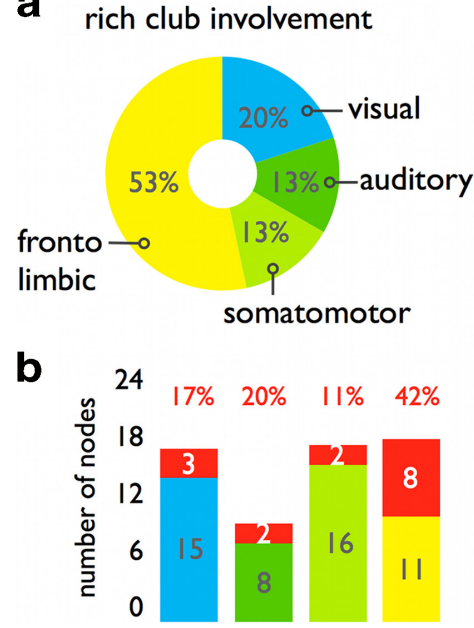

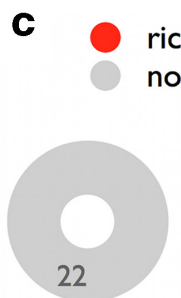

rich club non-rich club

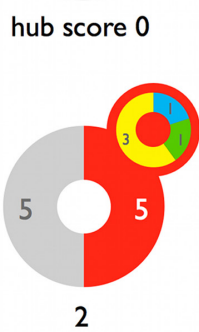

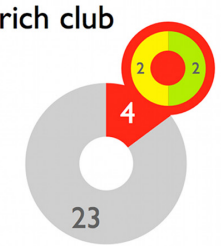

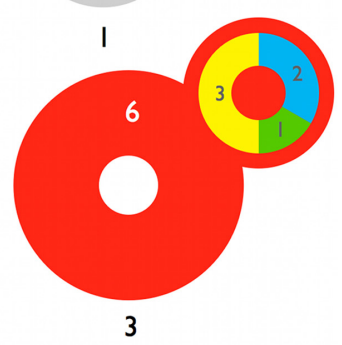

Figure 2. $\quad \boldsymbol{a}$, Rich club nodes were found to be present in all four functional modules. The pie graph shows how the 15 rich club nodes were distributed across the four modules. $\boldsymbol{b}$, Number of nodes that is involved in the rich club per functional module. $\boldsymbol{c}$, Distribution of rich club nodes (red) and non-rich club nodes (gray) across ranking scores (ranging from 0 to 3, top left to bottom right), expressing the number of times a node scored among the top 15 nodes on in-degree and out-degree $z_{i}$ and $P_{i}$ in the network. Rich club nodes were found to be well represented among the highest scoring nodes (class 3, labeled intermodule hubs), underscoring the central role of rich club hubs in linking all four functional communities of the network.

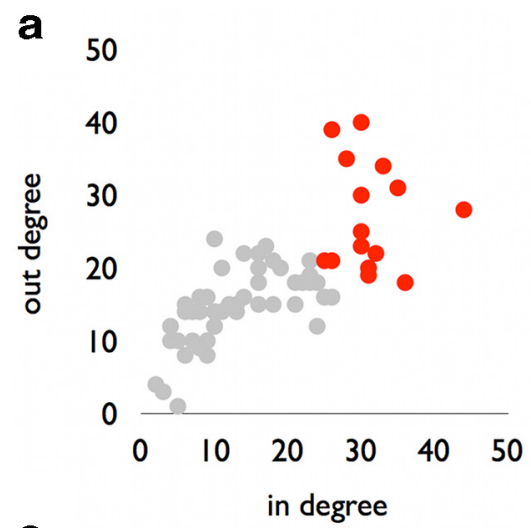

C
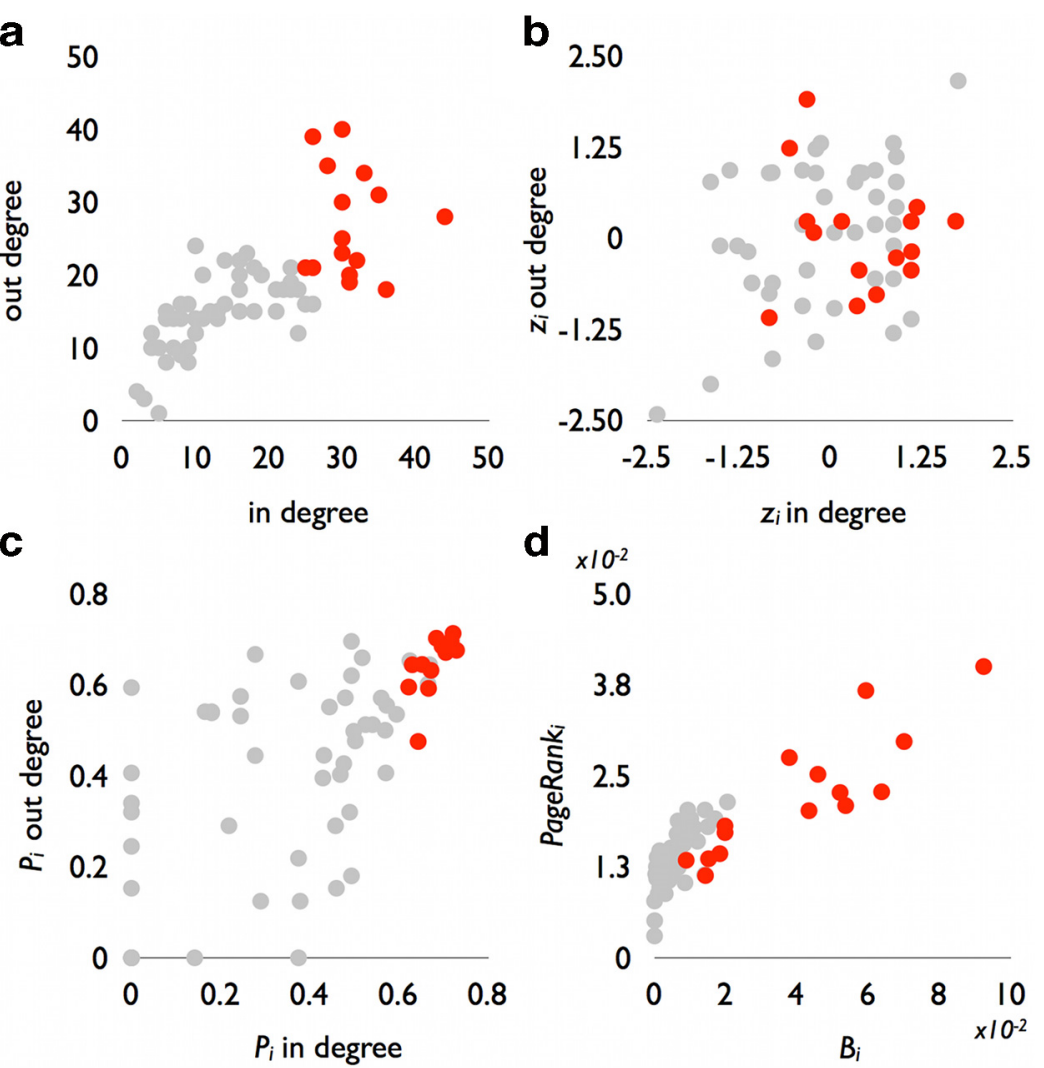

Figure 3. Node values and interactions of: $\boldsymbol{a}$, in-degree and out-degree; $\boldsymbol{b}$, in-degree and out-degree $z_{i}$; $\boldsymbol{c}$, in-degree and out-degree $P_{i} ; \boldsymbol{d}, B_{i}$ and PageRank ${ }_{i}$. Non-rich club nodes are shown in gray, and rich club nodes are shown in red.

\section{Rich club involvement in overlapping communities}

Using an information theoretic fuzzy community approach (Esquivel and Rosvall, 2011), regions of possible overlap between the four functional modules were identified by allowing nodes to participate in multiple communities while preserving their original module assignment. In the overlapping community pattern (Fig. 4a), rich club nodes were on average involved in 1.87 commu- nities (SD of 1.06), whereas non-rich club nodes were on average involved in 1.16 communities (SD of 0.37 ), significantly less than rich club regions $(p=0.0014 ; 10,000$ permutations). Furthermore, 7 (46\%) of 15 rich club nodes (including areas from all four functional domains) were involved in multiple communities (13\% participating in two communities, $27 \%$ in three communities, and $6 \%$ in all four communities), in contrast to only $16 \%$ of the non-rich club nodes. Such a diverse community involvement of rich club regions and their specific representation in areas in which multiple functional domains fuse together emphasizes the potential role of the rich club in interlinking different functional communities of the brain. Indeed, examining the number of functional communities to which each node directly connects (referred to as the "functional neighborhood" of a node) revealed that all rich club nodes are directly connected to nodes in all four functional communities (mean $\pm \mathrm{SD}, 4 \pm 0$ ), thus showing a significantly more diverse functional neighborhood compared with non-rich club nodes $(3 \pm 0.9 ; p<0.001$; 10,000 permutations) (Fig. $4 b$ ).

\section{Characterization of rich club, feeder, and local connections}

Figure $5 a$ shows the (weighted) connectivity matrix of the cat connectome, as adopted from Scannell et al. (1995). Classification of the nodes into rich club and non-rich club nodes allowed for the categorization of the connections of the network into three classes: (1) rich club connections, linking rich club members; (2) feeder connections, linking non-rich club nodes to rich club nodes; and (3) local connections, linking non-rich club nodes (Fig. 5b). Furthermore, information on the direction of projection pathways allowed for an additional classification of the feeder connections into feeder-in connections (those connections that project from non-rich club to rich club nodes) and feeder-out connections (those axonal projections that project from rich club nodes to non-rich club nodes). A circular graph representation of the cat connectome is given in Figure 6, showing rich club (red), feeder (orange), and local (yellow) connections. Richclub connections accounted for $14 \%$ of all traced connections of the cat cortex, feeder-in connections for $27 \%$, and feederout connections for $21 \%$ (together, $48 \%$ of the network). Local connections accounted for the remaining $38 \%$ of the total number of connections (Fig. 5c).

\section{Intermodule and intramodule connections}

A total of $43 \%$ of the connections were found to be intermodular, linking nodes of different functional modules, and $57 \%$ were 


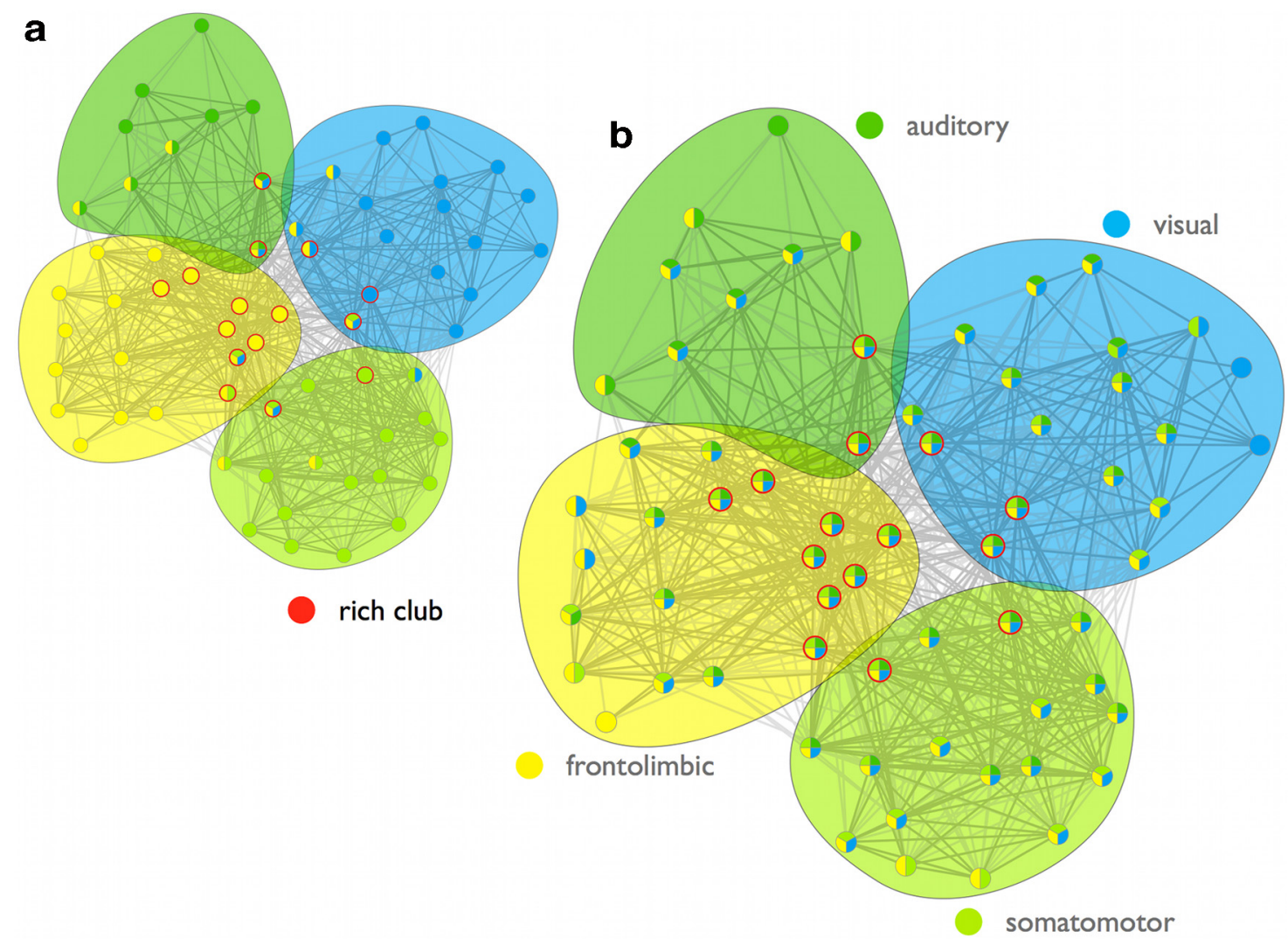

Figure 4. $a$, Overlap between the four functional domains of the cat brain as revealed by an information theoretic fuzzy community approach, allowing nodes of the network to participate in multiple communities while preserving their original community assignment (see Materials and Methods). Figure illustrates that rich club nodes are well represented among nodes that participate in multiple communities. $\boldsymbol{b}$, Functional neighborhood score of each node of the cat connectome, reflecting the number of functional communities to which each node is directly connected. All rich club nodes revealed connections to all four functional domains. Interestingly, although significantly less than rich club members, non-rich club nodes still displayed a remarkable high functional neighborhood score, with most of the nodes showing direct connections to other functional communities.

found to be intramodular, linking nodes of the same module. Of all intermodule connections, $86 \%$ was found to comprise rich club (22\%) and feeder (64\%) connections, underscoring an important role for these connections in intermodule communication (Fig. 5 c). Indeed, $67 \%$ of the rich club connections and $57 \%$ of the feeder connections were found to link nodes of different modules (Fig. 7a). When examining the number of connections per node, on average almost $39 \%$ of the connections of a node were found to be intermodular, and (on average) $61 \%$ of its connections were found to be intramodular. For rich club nodes, $60 \%$ of the connections were found to be intermodule connections (ranging from 38 to $90 \%$ ), whereas $32 \%$ of the connections of non-rich club nodes were intermodular (ranging from 0 to $80 \%$ ). Furthermore, $>60 \%$ of all non-rich club nodes had $>33 \%$ intermodule connections.

\section{Directionality}

A total of $72 \%$ of the total collection of connections was found to be bidirectional, and $28 \%$ of the structural projections were unidirectional (Fig. $7 b$ ). The majority of rich club connections were found to be bidirectional projections ( $86 \%$ bidirectional vs $14 \%$ unidirectional), more than feeder (69 vs 31\%) and local (70 vs $30 \%$ ) connections. In addition, feeder-out edges were found to be more often bidirectional ( $78 \%$ bidirectional vs $22 \%$ unidirectional) than feeder-in edges (62\% bidirectional vs 38\% unidirectional) (Fig. $7 c$ ). Intramodule connections were found to be predominantly bidirectional (79\% bidirectional vs $21 \%$ unidi- rectional), more pronounced than intermodule connections (62\% bidirectional vs $38 \%$ unidirectional).

\section{Structural homogeneity of connections}

Measuring the structural homogeneity of each connection in the network, expressed as the overlap $O$ between the (one-step) connection neighborhoods of the two nodes joined by the connection, revealed a significantly lower overlap in the connection neighborhoods of feeder connections (mean $\pm \mathrm{SD}, 0.37 \pm 0.13$ ) compared with rich club $(0.49 \pm 0.11 ; p<0.001)$ and local $(0.49 \pm 0.16 ; p<0.001 ; 10,000$ permutations $)$ connections. No differences in $O$ values between feeder-in and feeder-out connections were observed.

\section{Module diversity of connections}

In addition to the structural homogeneity, the module diversity of each connection was also computed, examining the richness of the functional neighborhoods of its endpoints (see Materials and Methods). Rich club connections (mean \pm SD, $1 \pm 0$ ) showed a higher module diversity compared with feeder $(0.82 \pm 0.19 ; p<0.001)$ and local $(0.66 \pm 0.24 ; p<0.001)$ connections. Bidirectional links (mean $\pm \mathrm{SD}, 0.80 \pm 0.23$ ) were found to have a higher diversity score than unidirectional links, but this effect was only marginally significant $(0.77 \pm$ $0.23 ; p=0.05 ; 10,000$ permutations). 


\section{a structural connections}

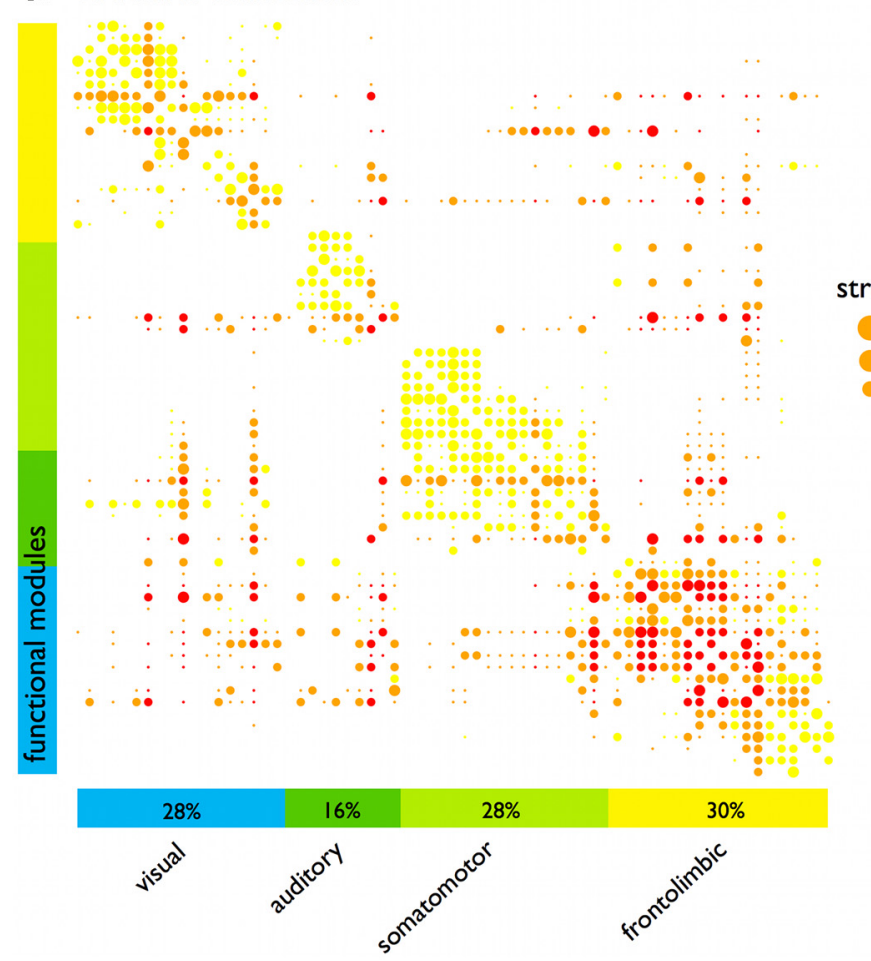

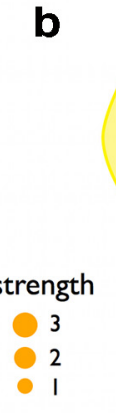

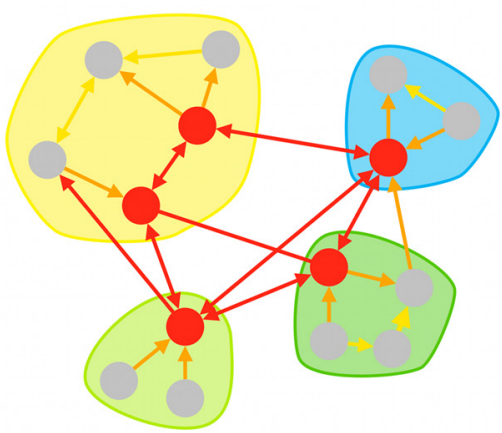

C
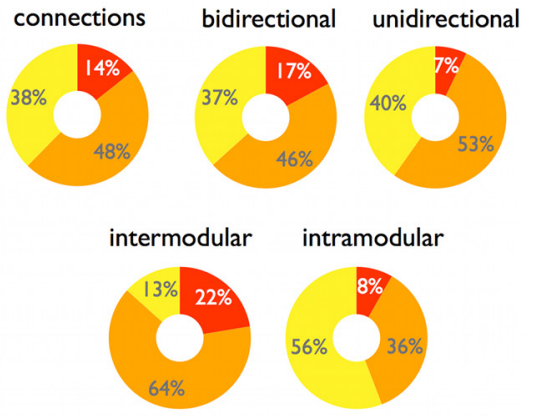

Figure 5. a, Connectivity matrix of the cat connectome, with nodes ordered according to the participation in the four functional modules. Connections are color coded according to their connection class: rich club (red), feeder (orange), or local (yellow). $\boldsymbol{b}$, Schematic figure of the rich club (red) and non-rich club (gray) nodes and directional rich club (red), feeder (orange), and local (yellow) connections. c, Metrics of interest of the three classes of connections. Examined metrics include the number of connections, distribution of bidirectional and unidirectional connections, and number of intermodule and intramodule connections.

\section{Path motifs}

Tracing all shortest communication paths in the network that traveled between two non-rich club nodes of different functional communities revealed that $89.5 \%$ of all paths traveled through a rich club node (significantly more than in randomized networks; $p<0.001)$, with $24.2 \%(p<0.001)$ traveling through at least one rich club connection. These values were found to be much higher than in a set of comparable random networks, in which $65 \%$ of the intermodule communications paths crossed a rich club node (SD, 1.4\%) and $8.9 \%$ of the paths crossed at least one rich club connection (SD, 1.1\%) (1000 random networks examined). Examining the ordered sequence of connections-referred to as path motifs-passed when traveling along these shortest communication paths in the network revealed a strong prevalence of paths that passed through rich club connections, following a F-RC-F path motif ( $23 \%$ of all shortest paths, $2.6 \times$ higher than random; $p<0.001 ; 1000$ random networks) (Fig. 8b). Note that, because of a relative low spatial resolution, the overall shortest path length in the cat dataset is low ( $97 \%$ of intermodule paths consist of just two or three links, and paths of length five are entirely absent), making long motifs, such as L-F-RC-F-L as reported in human (van den Heuvel et al., 2012) and macaque (Harriger et al., 2012) brain impossible to occur. For intermodule paths, the F-RC-F motif is the only submotif of L-F-RC-F-L of less than four steps and therefore its natural analog in the cat connectome. In contrast, paths that followed an F-F motif were found to be less prevalent in the cat brain compared with random networks (22.3 vs $38.7 \%$ in random networks; Fig. $8 b$ ). Also note the relative high occurrence of paths following an L-F-F motif $(p<0.001$; Fig. $8 b)$, representing paths that directly pass through a rich club node without traversing over a rich club connection when traveling between nodes of different modules, indicating an important role for feeder connections in intermodule communication.

Further delineating paths that followed the most prevalent F-RC-F path motif (Fig. 8c), now including information on the intermodular and intramodular character of the connections of the network, revealed an above-chance level occurrence of paths that followed a "feeder-in-intramodule to rich club-intermodule to feeder-out-intramodule" motif ( 15 times higher than random; $p<0.001$ ). This was higher than (for example) paths that followed a "feeder-in-intermodule to rich club-intermodule to feeder-out-intramodule" $(4.7 \times$ more than random; $p<0.001)$ or a "feeder-in-intermodule to rich club-intramodule to feederout-intramodule" motif (not significantly different from random; Fig. 8c). Interestingly, consistent with the results shown in Figure $8 b$, paths that followed a "local-intramodule to feederintermodule to feeder-intramodule" motif were found to be among the highest occurring motifs in the network $(p<0.001$; Fig. $8 c$ ), suggesting that, besides intermodule rich club connections, feeder-in and feeder-out intermodule connections (linking non-rich club nodes to rich club nodes) play an important role in maintaining short communication pathways between different functional modules in the cat brain.

\section{Discussion}

This study provides additional support for the rich club of the brain to form an infrastructure for communication between different domains of the mammalian brain. Extending recent reports of rich club formation in the C. elegans (Towlson et al., 2013), cat (Zamora-López et al., 2009), macaque (Harriger et al., 2012), and human (van den Heuvel and Sporns, 2011; Collin et 


\section{a}

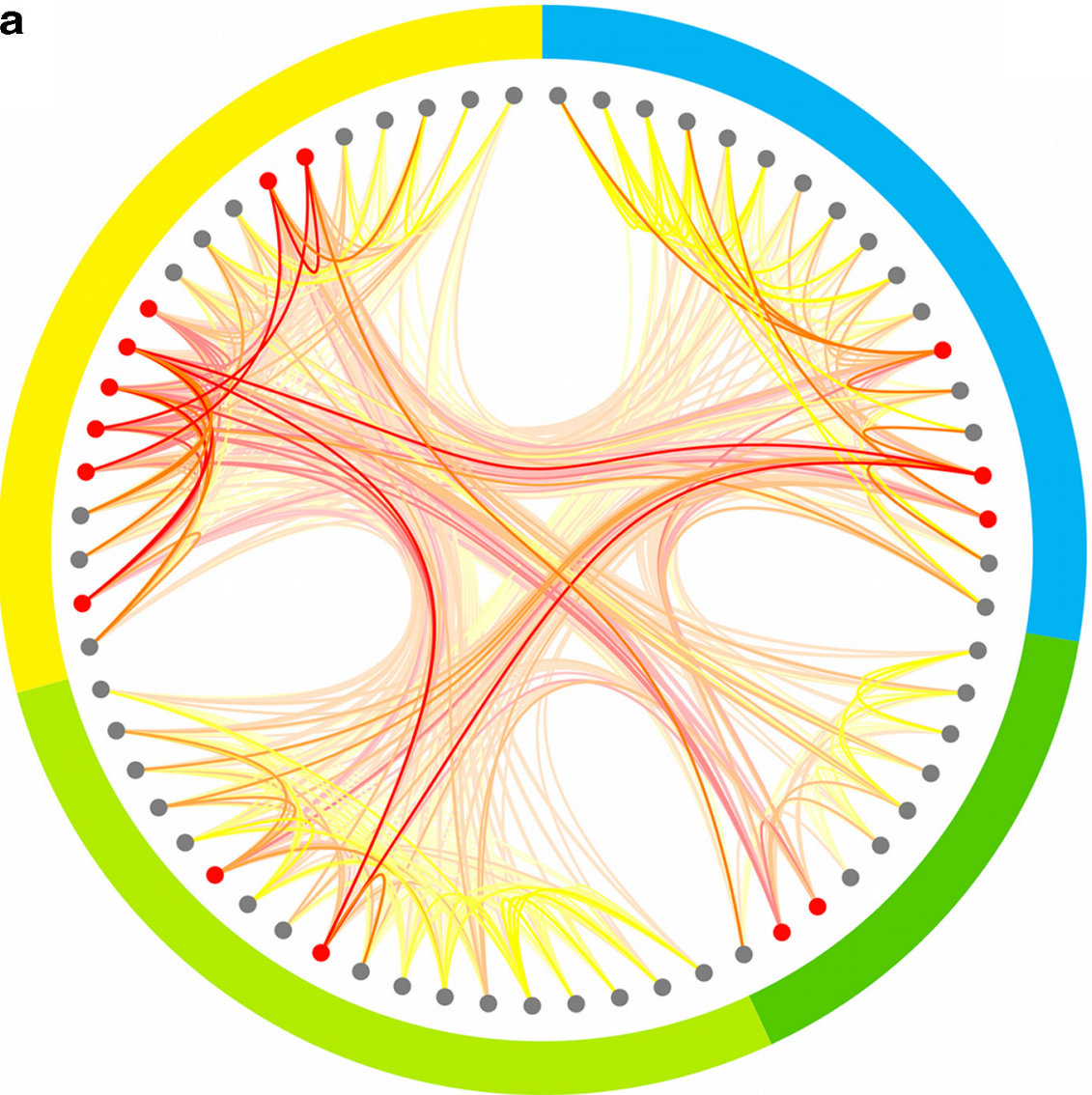

b

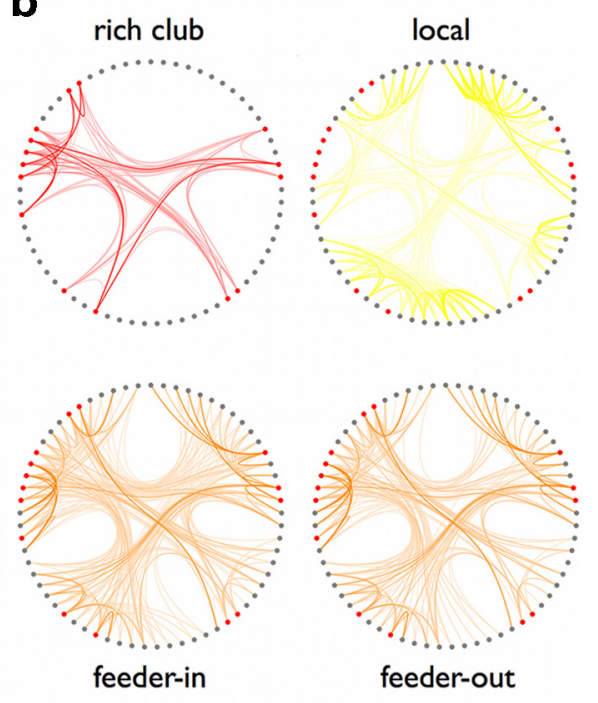

rich club

feeder

local

rich club

non-rich club

Figure 6. $\boldsymbol{a}$, Ring plot of the cat connectome. Nodes of the cat brain are ordered on a ring according to their module participation. Connections are colored according to their connection class. $\boldsymbol{b}$, Connections of the network split in rich club, local, and feeder-in and feeder-out connections. Nodes in the plots of $\boldsymbol{b}$ are arranged in the same order as in $\boldsymbol{a}$. The figure shows a clear intermodular character of rich club and feeder connections, whereas local connections mostly connect nodes within the same module.

a

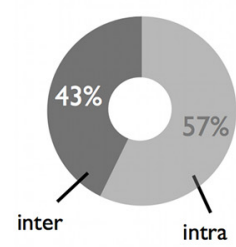

b

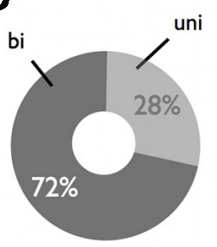

inter / intramodular
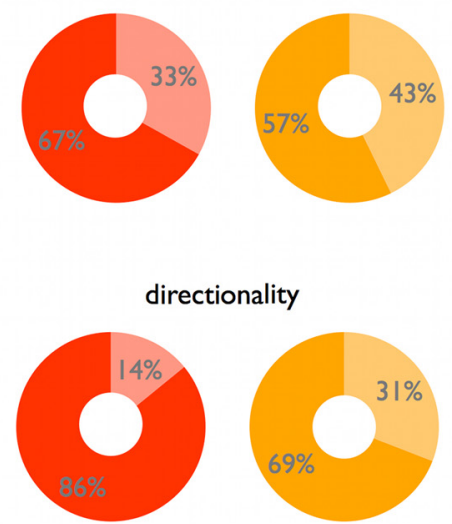

directionality

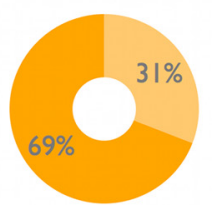

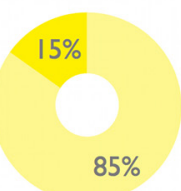

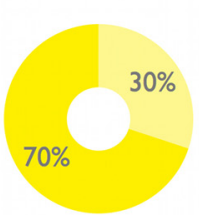

C

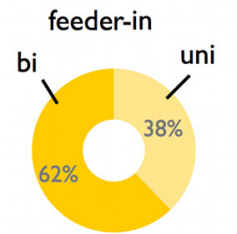

feeder-out

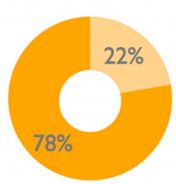

inter
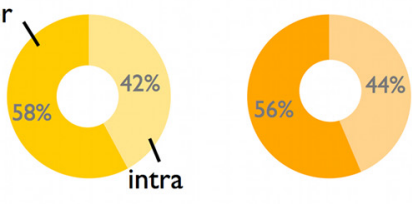

Figure 7. $\boldsymbol{a}$, Distribution of intermodule and intramodule connections across all connections (gray) and across the class of rich club (red), feeder (orange), and local (yellow) connections. $\boldsymbol{b}$, Distribution of bidirectional (bi) and unidirectional (uni) connections across all connections, and across the three classes of connections. c, Distribution of bidirectional (bi) and unidirectional (uni) connections and intermodule and intramodule connections across feeder-in (light orange) and feeder-out (dark orange) connections.

al., 2013) cortex, our findings provide six lines of evidence to pinpoint the rich club as a central anatomical infrastructure to interconnect functional domains of the mammalian brain.

First, analyzing data of the macroscopic cat connectome as presented in the seminal work of Scannell et al. (1995) almost 20 years ago, rich club nodes were found to be present in all four functional modules of the cat brain. This observation indicates that the rich club involves regions from all functional domains, including not only frontolimbic, but also primary visual, auditory, and somatomotor networks. Second, rich club nodes were found to show a particular diverse connectivity profile, being well represented among the so-called intermodule hubs of the network. Third, more than two-thirds of all rich club and more than half of all feeder connections were found to span between different functional modules, indicating a clear prevalence of these types of connections to bridge different functional communities. 
a

b
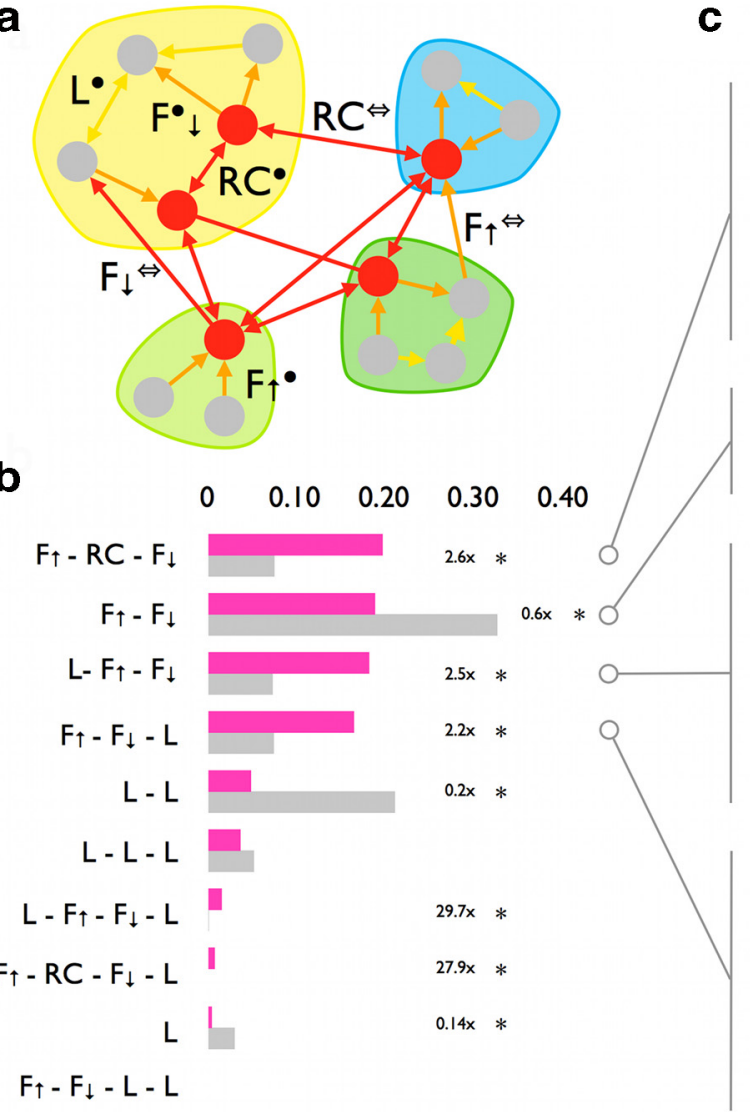

C

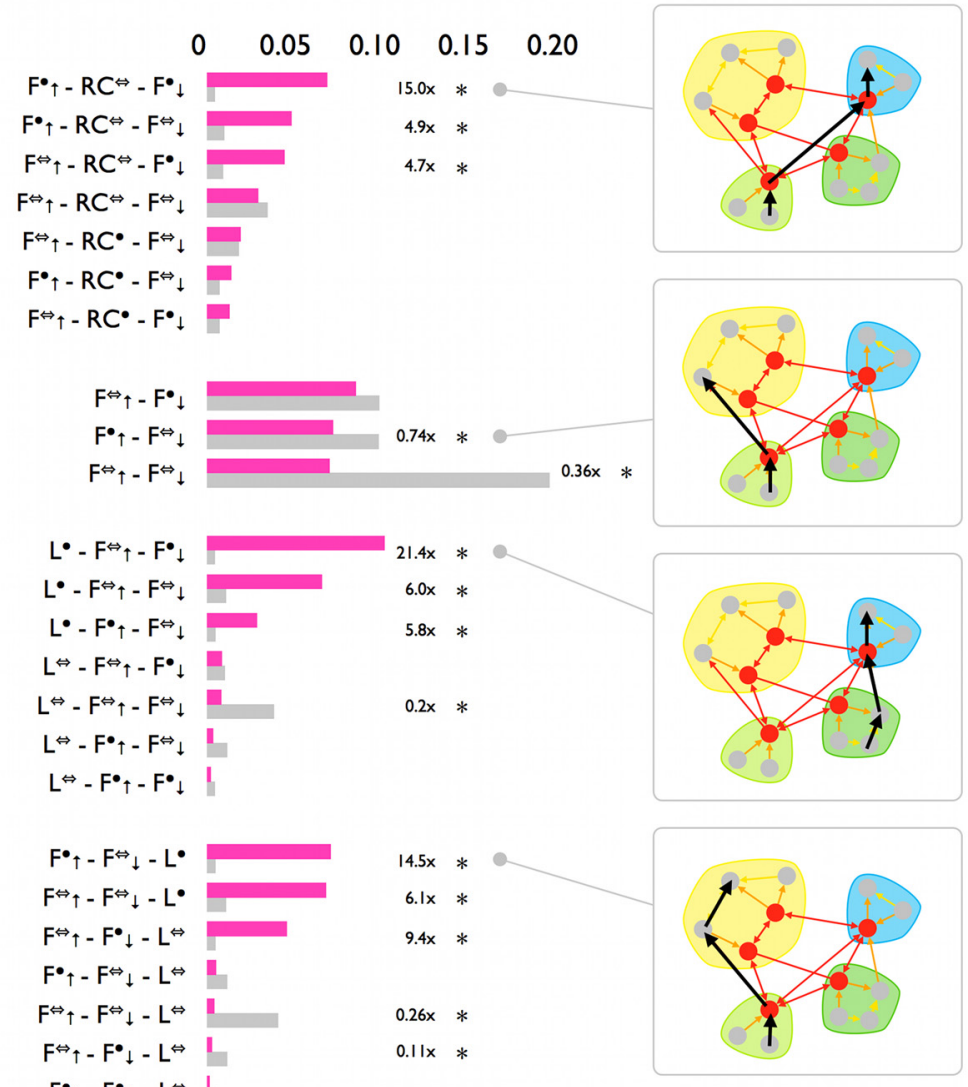

Figure 8. a shows a schematic figure of a network with rich club organization, indicating eight classes of connections: $\mathrm{RC} \Leftrightarrow$, rich club intermodular; $\mathrm{RC}^{\bullet}$, rich club intramodular; $\mathrm{F} \Leftrightarrow$, feeder-out intermodular; $\mathrm{F} \Leftrightarrow \Leftrightarrow_{\downarrow}$, feeder-in intermodular; $\mathrm{F}_{\downarrow}{ }_{\downarrow}$, feeder-out intramodular; $\mathrm{F}_{\uparrow}^{\bullet}$, feeder-in intramodular; $\mathrm{L} \Leftrightarrow$, local intermodular; $\mathrm{L}^{\bullet}$, local intramodular. $\boldsymbol{b}$, Distribution of path motifs of all shortest paths between local nodes in different functional communities, taking into account information on rich club, feeder-in, feeder-out, and local connections. Path motif counts of the cat dataset are depicted in pink, and path motif counts in a random situation (average across 1000 random networks) are depicted in gray. Figure illustrates that the F-RC-F motif occurs $2.6 \times$ more in the cat dataset compared with the random model $(p<0.001)$. $c$, Distribution of path motifs as shown in $\boldsymbol{b}$ now further specified including information on the intramodular or intermodular character of the passed connections. c shows the path motif counts of the four highest scoring path motifs of $\boldsymbol{b}$. ${ }^{*} p<0.05$, Bonferroni's corrected.
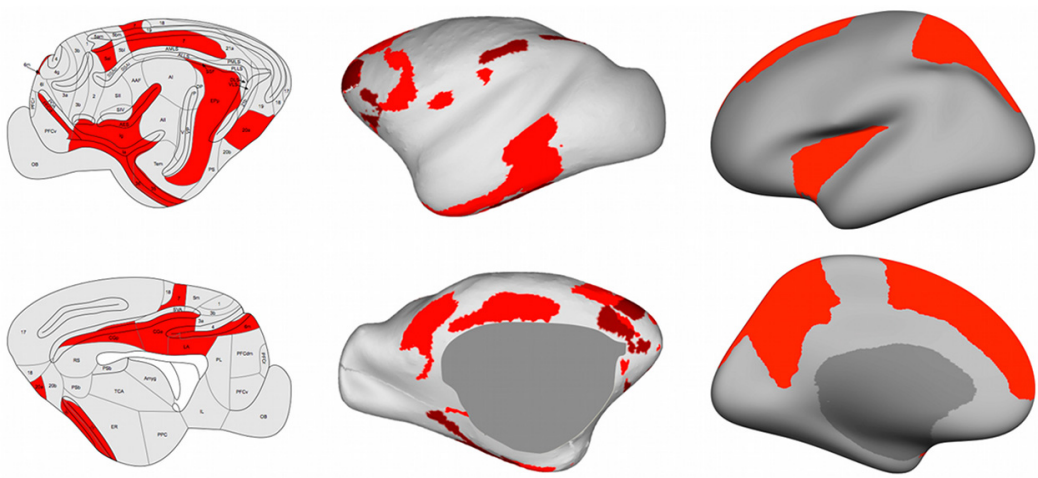

cat

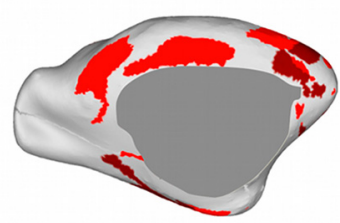

macaque

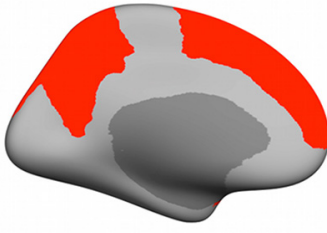

human

Figure 9. Cortical rich clubs across species. From left to right, figures show rich club regions color coded in red of the cat cortex (as presented by Zamora-López et al., 2009, 2011 and the present paper), macaque cortex (figure adapted from Harriger et al., 2012), and human cortex (low resolution, 34 cortical nodes per hemisphere; data from van den Heuvel and Sporns, 2011). Overlapping with rich club involvement in the cat cortex as presented in this paper, the macaque (Harriger et al., 2012) and human (van den Heuvel et al., 2012) rich club have also been reported to involve occipital and parietal visual and sensory regions, temporal auditory regions, frontal (pre)motor regions, as well as insular, medioparietal, and mediofrontal regions overlapping the limbic system.

This stands in contrast to local connections of which the vast majority (84\%) connected nodes of the same module. Fourth, all rich club nodes were found to be directly connected to nodes across all four functional domains. Fifth, an information theo- retic approach to detect possible overlap between the four functional modules (Esquivel and Rosvall, 2011) revealed a clear multimodal character of rich club nodes, suggesting that rich club nodes operate at the topological "border zones" of functional subsystems (Fig. 4). Sixth, examination of the sequence of connection classes along communication paths in the brain showed a clear participation of the rich club in intermodule paths, suggesting an important role of rich club and feeder connections in intermodule communication (Fig. 8).

Connections linked to rich club members were found to be well represented among intermodule connections. Although, split up by connection class, richclub connections showed the highest proportion of intermodule connections with respect to the overall count of their class (Fig. 7), both feeder-in and feederout connections were also found to be well represented among intermodule connections and intermodule paths. More than half of all feeder connections were found to link nodes of different functional modules (Fig. 7), and paths involv- 
ing feeder connections were found to be among the highest occurring path motifs (in particular, the $\mathrm{L}^{\bullet}-\mathrm{F}^{\Leftrightarrow} \Leftrightarrow-\mathrm{F}_{\downarrow}^{\bullet}$ motif; see Fig. $8 C$ ) in the network. This suggests that feeder connections are likely to play a crucial role in maintaining high levels of direct connectivity between functional domains and that rich club connections may not necessarily be designed to only facilitate quick global communication routes. Rich club nodes and connections showed a particular high level of module diversity, suggesting that they are positioned in areas in which information from many functional modules passes and merges. With communication in neural systems involving continuous transformation and integration, the diverse character of rich club nodes and their edges thus leaves room for the hypothesis that the rich club does not only comprise a set of global communication pathways but may constitute, as a core system, a central place for global integration of information. Theories of consciousness and cognition have suggested that global integration of information may take place in a "global workspace," hypothesized as a dynamical core in which segregated functional systems can share their information though means of neuronal interactions (Dehaene and Naccache, 2001). The rich club system connects a large proportion of all central hubs of the network and participates in a large proportion of global communication pathways, making it an ideal candidate for a neural substrate of an integrative global workspace in the mammalian brain (Harriger et al., 2012).

The observed rich club topology of the cat cortex is consistent with recently reported rich club organization of the macaque and human cortex (Harriger et al., 2012; Shen et al., 2012; van den Heuvel et al., 2012). Although caution is needed when directly comparing brain regions across species (Sereno and Tootell, 2005), the macaque and human rich club are also suggested to involve occipital and parietal visual and sensory regions, temporofrontal insular regions, frontal motor and premotor regions, and medioparietal and mediofrontal regions (Fig. 9). The spatial and topological overlap of rich club systems across the cat, macaque, and human cortex proposes a strong preservation of this subsystem across species, suggesting high value of retaining a neural substrate for integration of information between separated systems in the brain.

At least one additional observation stood out and deserves a more detailed discussion. Besides nodes of the rich club, the rest of the network was also found to show a distinct intermodular character. Indeed, almost half of all macroscopic white matter projections in the cat brain were found to link nodes of different functional modules. Examining the placement of connections per node, on average $30 \%$ of the edges of non-rich club nodes were found to directly connect to a node in one of the other functional domains, among which a large proportion of feeder connections. More than $60 \%$ of all peripheral non-rich club nodes was found to show a relatively diverse character, having $>33 \%$ of their connections projecting directly to or from other functional domains. These observations tend to suggest that the mammalian brain on the macroscopic level has a less modular and perhaps more integrative organization than commonly argued. Such a diverse character of the majority of brain regions in the brain is supported by functional studies of the human brain, suggesting a dynamic participation of cortical areas into multiple communities (Bassett et al., 2006), as well as the observation that most cortical areas show involvement in a wide variety of cognitive tasks (Anderson and Pessoa, 2011).

Some comments are in place when interpreting the findings of this study. First, in the main analysis of this study, no information on the strength of corticocortical projections was taken into ac- count. Including information from the original classification of connections into "weak," "medium," and "strong" connections did not change the nature of our findings, nor did it reveal clear distinctions in connectivity strength between connection classes. However, information available on the strength of corticocortical projections in the cat connectome dataset is coarse (Scannell et al., 1995), and future studies examining the architecture of tractbased connectomes including more direct and more extensive information on connectivity strength (Markov et al., 2012) would be of high interest. Second, in contrast to aforementioned reports of rich club formation in the human brain, the cat dataset comprises information from a single hemisphere only, which is likely to have an impact on the path motif analysis as only intrahemispheric paths can be examined. However, the considerable overlap between the rich club in the human brain and the cat and macaque brain (Harriger et al., 2012) (with the latter also based on single hemisphere data) further supports the notion that the rich club does not merely relate to the bridging of two hemispheres but rather to linking functional domains in general. Third, the cat connectome, as well as the macaque connectome based on the CoCoMac (for Collation of Connectivity Data on the Macaque Brain) database (Stephan et al., 2001) and the original reconstruction of the connectome of the Caenorhabditis elegans (White et al., 1986), are not the result of connectivity mapping within a single animal but rather involve a reconstruction of the connectome based on the aggregation of connectivity data form a large number of (mostly) single injection site and single connection studies. This in contrast to in vivo diffusionbased studies of the human connectome, which do allow for the examination of brain networks as reconstructed from single individuals (Hagmann et al., 2008; Iturria-Medina et al., 2008; Fornito et al., 2011; van den Heuvel and Sporns, 2011; Zalesky et al., 2011). Fourth, a number of analyses in this paper assume an important role for shortest paths in interactions between brain regions. However, as noted previously (van den Heuvel et al., 2012), the human brain is likely a system in which its elements only have access to information about the local structure of the network and are thus unable to select shortest paths on basis of knowledge about the global architecture of the network. Recent studies have proposed a number of interesting metrics that express communication in networks in terms of diffusion-like processes (Esquivel and Rosvall, 2011; Goñi et al., 2013), and studies examining the notion of path motifs in combination with these diffusion-based metrics would be of particular interest.

Our findings show an important role for the rich club to crosslink different functional communities in the cat brain. A central bridge function of rich club and feeder connections further supports the theory of the rich club to form a key system for global communication and integration in the mammalian brain.

\section{References}

Anderson LM, Pessoa L (2011) Quantifying the diversity of neural activations in individual brain regions. Proceedings of the 33rd Annual Conference of the Cognitive Science Society, Boston, MA, July.

Bassett DS, Meyer-Lindenberg A, Achard S, Duke T, Bullmore E (2006) Adaptive reconfiguration of fractal small-world human brain functional networks. Proc Natl Acad Sci U S A 103:19518-19523. CrossRef Medline

Bialonski S, Wendler M, Lehnertz K (2011) Unraveling spurious properties of interaction networks with tailored random networks. PLoS One 6:e22826. CrossRef Medline

Buckner RL, Sepulcre J, Talukdar T, Krienen FM, Liu H, Hedden T, AndrewsHanna JR, Sperling RA, Johnson KA (2009) Cortical hubs revealed by intrinsic functional connectivity: mapping, assessment of stability, and relation to Alzheimer's disease. J Neurosci 29:1860-1873. CrossRef Medline 
Bullmore E, Sporns O (2009) Complex brain networks: graph theoretical analysis of structural and functional systems. Nat Rev Neurosci 10:186198. CrossRef Medline

Bullmore E, Sporns O (2012) The economy of brain network organization. Nat Rev Neurosci 13:336-349. CrossRef Medline

Colizza V, Flammini A, Serrano MA, Vespignani A (2006) Detecting rich club ordering in complex networks. Nat Phys 2:110-115. CrossRef

Collin G, Sporns O, Mandl RC, Van den Heuvel MP (2013) Structural and functional aspects relating to cost and benefit of rich club organization in the human cerebral cortex. Cereb Cortex. Advance online publication. Retrieved June 10, 2013. doi:10.1093/cercor/bht064. CrossRef Medline

Dehaene S, Naccache L (2001) Towards a cognitive neuroscience of consciousness: basic evidence and a workspace framework. Cognition 79:137. CrossRef Medline

Esquivel AV, Rosvall M (2011) Compression of flow can reveal overlappingmodule organization in networks. Phys Rev E 1:021025. CrossRef Medline

Felleman DJ, Van Essen DC (1991) Distributed hierarchical processing in the primate cerebral cortex. Cereb Cortex 1:1-47. CrossRef Medline

Fornito A, Zalesky A, Bassett DS, Meunier D, Ellison-Wright I, Yücel M, Wood SJ, Shaw K, O’Connor J, Nertney D, Mowry BJ, Pantelis C, Bullmore ET (2011) Genetic influences on cost-efficient organization of human cortical functional networks. J Neurosci 31:3261-3270. CrossRef Medline

Fornito A, Harrison BJ, Zalesky A, Simons JS (2012) Competitive and cooperative dynamics of large-scale brain functional networks supporting recollection. Proc Natl Acad Sci U S A 109:12788-12793. CrossRef Medline

Goñi J, Avena-Koenigsberger A, Velez de Mendizabal N, van den Heuvel MP, Betzel RF, Sporns O (2013) Exploring the morphospace of communication efficiency in complex networks. PLoS One 8:e58070. CrossRef Medline

Guimerà R, Nunes Amaral LA (2005) Functional cartography of complex metabolic networks. Nature 433:895-900. CrossRef Medline

Hagmann P, Cammoun L, Gigandet X, Meuli R, Honey CJ, Wedeen VJ, Sporns O (2008) Mapping the structural core of human cerebral cortex. PLoS Biol 6:e159. CrossRef Medline

Harriger L, van den Heuvel MP, Sporns O (2012) Rich club organization of macaque cerebral cortex and its role in network communication. PLoS One 7:e46497. CrossRef Medline

Iturria-Medina Y, Sotero RC, Canales-Rodríguez EJ, Alemán-Gómez Y, Melie-García L (2008) Studying the human brain anatomical network via diffusion-weighted MRI and Graph Theory. Neuroimage 40:1064-1076. CrossRef Medline

Markov NT, Ercsey-Ravasz MM, Ribeiro Gomes AR, Lamy C, Magrou L, Vezoli J, Misery P, Falchier A, Quilodran R, Gariel MA, Sallet J, Gamanut R, Huissoud C, Clavagnier S, Giroud P, Sappey-Marinier D, Barone P, Dehay C, Toroczkai Z, Knoblauch K, Van Essen DC, Kennedy H (2012) A weighted and directed interareal connectivity matrix for macaque cerebral cortex. Cereb Cortex. Advance online publication. Retrieved June 10, 2013. doi:10.1093/cercor/bhs270. CrossRef Medline

Maslov S, Sneppen K (2002) Specificity and stability in topology of protein networks. Science 296:910-913. CrossRef Medline

Newman ME (2006) Modularity and community structure in networks. Proc Natl Acad Sci U S A 103:8577-8582. CrossRef Medline

Page L, Brin S, Motwani R, Winograd T (1999) The PageRank citation ranking: bringing order to the web. Technical Report 1999-66. Stanford, CA: Stanford University.

Rubinov M, Sporns O (2010) Complex network measures of brain connectivity: uses and interpretations. Neuroimage 52:1059-1069. CrossRef Medline

Scannell JW, Young MP (1993) The connectional organization of neural systems in the cat cerebral cortex. Curr Biol 3:191-200. CrossRef Medline
Scannell JW, Blakemore C, Young MP (1995) Analysis of connectivity in the cat cerebral cortex. J Neurosci 15:1463-1483. Medline

Selemon LD, Goldman-Rakic PS (1988) Common cortical and subcortical targets of the dorsolateral prefrontal and posterior parietal cortices in the rhesus monkey: evidence for a distributed neural network subserving spatially guided behavior. J Neurosci 8:4049-4068. Medline

Sepulcre J, Sabuncu MR, Johnson KA (2012) Network assemblies in the functional brain. Curr Opin Neurol 25:384-391. CrossRef Medline

Sereno MI, Tootell RB (2005) From monkeys to humans: what do we now know about brain homologies? Curr Opin Neurobiol 15:135-144. CrossRef Medline

Shen K, Bezgin G, Hutchison RM, Gati JS, Menon RS, Everling S, McIntosh AR (2012) Information processing architecture of functionally defined clusters in the macaque cortex. J Neurosci 32:17465-17476. CrossRef Medline

Sporns O (2011) The human connectome: a complex network. Ann N Y Acad Sci 1224:109-125. CrossRef Medline

Sporns O, Tononi G, Kötter R (2005) The human connectome: a structural description of the human brain. PLoS Comput Biol 1:e42. CrossRef Medline

Sporns O, Honey CJ, Kötter R (2007) Identification and classification of hubs in brain networks. PLoS One 2:e1049. CrossRef Medline

Stephan KE, Kamper L, Bozkurt A, Burns GA, Young MP, Kötter R (2001) Advanced database methodology for the Collation of Connectivity data on the Macaque brain (CoCoMac). Philos Trans R Soc Lond B Biol Sci 356:1159-1186. CrossRef Medline

Tomasi D, Volkow ND (2010) Functional connectivity density mapping. Proc Natl Acad Sci U S A 107:9885-9890. CrossRef Medline

Towlson EK, Vértes PE, Ahnert SE, Schafer WR, Bullmore ET (2013) The rich club of the C. elegans neuronal connectome. J Neurosci 33:63806387. CrossRef Medline

van den Heuvel MP, Hulshoff Pol HE (2010) Exploring the brain network: a review on resting-state fMRI functional connectivity. Eur Neuropsychopharmacol 20:519-534. CrossRef Medline

van den Heuvel MP, Sporns O (2011) Rich-club organization of the human connectome. J Neurosci 31:15775-15786. CrossRef Medline

van den Heuvel MP, Kahn RS, Goñi J, Sporns O (2012) High cost, high capacity backbone for global brain communication. Proc Natl Acad Sci U S A 109:11372-11377. CrossRef Medline

Van den Heuvel MP, Sporns O, Collin G, Scheeuwe T, Mandl RCW, Cahn W, Goñi J, Hulshoff Pol HE, Kahn RS (2013) Abnormal rich club organization and functional brain dynamics in schizophrenia. JAMA Psychiatry $1-10$. CrossRef

White JG, Southgate E, Thomson JN, Brenner S (1986) The structure of the nervous system of the nematode Caenorhabditis elegans. Philos Trans R Soc Lond B Biol Sci 314:1-340. CrossRef Medline

Young MP (1993) The organization of neural systems in the primate cerebral cortex. Proc Biol Sci 252:13-18. CrossRef Medline

Zalesky A, Fornito A, Seal ML, Cocchi L, Westin CF, Bullmore ET, Egan GF, Pantelis C (2011) Disrupted axonal fiber connectivity in schizophrenia. Biol Psychiatry 69:80-89. CrossRef Medline

Zalesky A, Fornito A, Bullmore E (2012) On the use of correlation as a measure of network connectivity. Neuroimage 60:2096-2106. CrossRef Medline

Zamora-López G, Zhou C, Kurths J (2009) Graph analysis of cortical networks reveals complex anatomical communication substrate. Chaos 19: 015117. CrossRef Medline

Zamora-López G, Zhou C, Kurths J (2010) Cortical hubs form a module for multisensory integration on top of the hierarchy of cortical networks. Front Neuroinform 4:1. CrossRef Medline

Zamora-López G, Zhou C, Kurths J (2011) Exploring brain function from anatomical connectivity. Front Neurosci 5:83. CrossRef Medline 